\title{
Rice transposable elements are characterized by various methylation environments in the genome Miwako Takata ${ }^{\dagger 1}$, Akihiro Kiyohara ${ }^{\dagger 1}$, Atsuko Takasu ${ }^{\dagger 1}$, Yuji Kishima*1, Hisako Ohtsubo ${ }^{2}$ and Yoshio Sano ${ }^{2}$
}

\author{
Address: ${ }^{1}$ Laboratory of Plant Breeding, Graduate School of Agriculture, Hokkaido University, Sapporo 060-8589, Japan and ${ }^{2}$ Institute of \\ Molecular and Cellular Biosciences, University of Tokyo, Bunkyo-ku, Tokyo 113-0032, Japan \\ Email: Miwako Takata -miwako-takata@taiho.co.jp; Akihiro Kiyohara - moero-hanshin@ @otmail.co.jp; \\ Atsuko Takasu - oneone.nyan_nyan@r8.dion.ne.jp; Yuji Kishima* - kishima@abs.agr.hokudai.ac.jp; Hisako Ohtsubo - hohtsubo@iam.u- \\ tokyo.ac.jp; Yoshio Sano - rysano@abs.agr.hokudai.ac.jp \\ * Corresponding author †Equal contributors
}

Published: 20 December 2007

BMC Genomics 2007, 8:469 doi:10.1/86/|47|-2/64-8-469
Received: 3I May 2007

Accepted: 20 December 2007

This article is available from: http://www.biomedcentral.com/I47I-2/64/8/469

(c) 2007 Takata et al; licensee BioMed Central Ltd.

This is an Open Access article distributed under the terms of the Creative Commons Attribution License (http://creativecommons.org/licenses/by/2.0), which permits unrestricted use, distribution, and reproduction in any medium, provided the original work is properly cited.

\begin{abstract}
Background: Recent studies using high-throughput methods have revealed that transposable elements (TEs) are a comprehensive target for DNA methylation. However, the relationship between TEs and their genomic environment regarding methylation still remains unclear. The rice genome contains representatives of all known TE families with different characteristics of chromosomal distribution, structure, transposition, size, and copy number. Here we studied the DNA methylation state around 12 TEs in nine genomic DNAs from cultivated rice strains and their closely related wild strains.

Results: We employed a transposon display (TD) method to analyze the methylation environments in the genomes. The I2 TE families, consisting of four class I elements, seven class II elements, and one element of a different class, were differentially distributed in the rice chromosomes: some elements were concentrated in the centromeric or pericentromeric regions, but others were located in euchromatic regions. The TD analyses revealed that the TE families were embedded in flanking sequences with different methylation degrees. Each TE had flanking sequences with similar degrees of methylation among the nine rice strains. The class I elements tended to be present in highly methylated regions, while those of the class II elements showed widely varying degrees of methylation. In some TE families, the degrees of methylation were markedly lower than the average methylation state of the genome. In two families, dramatic changes of the methylation state occurred depending on the distance from the TE.

Conclusion: Our results demonstrate that the TE families in the rice genomes can be characterized by the methylation states of their surroundings. The copy number and degree of conservation of the TE family are not likely to be correlated with the degree of methylation. We discuss possible relationships between the methylation state of TEs and their surroundings. This is the first report demonstrating that TEs in the genome are associated with a particular methylation environment that is a feature of a given TE.
\end{abstract}




\section{Background}

Cytosine methylation is not only responsible for regulation of gene expression and immobilization of transposable elements (TEs) $[1,2]$, but also gives cues about genomic environments [3]. In eukaryotic chromosomes, condensed regions such as the centromere and heterochromatin have intensively accumulated TEs, resulting in heavy DNA methylation in organisms that possess methylation machinery [4]. Recent genome-wide analyses using high-throughput methods revealed that plant genomes show differential methylation states between genes and repetitive sequences: gene-rich regions are present in the hypomethylated fraction, while repetitive sequences are often found in the hypermethylated fraction of the genome [5-7]. A comparative study of methylation status between Arabidopsis ecotypes revealed that TEs are commonly methylated, while genic methylation is highly polymorphic [8]. On the other hand, in mammalian genomes, genes and repeats did not show differential DNA methylation states [9]. Every repetitive sequence has a unique set of features regarding structure, transpositional manner, size, copy number, etc. Tran et al. [10] reported that the major epigenetic systems (DNA methylation and histone H3 lysine-9) in the Arabidopsis genome target TEs in a genome-wide manner, irrespective of element type and position. However, plant genomes contain an enormous number of repetitive sequences that are widely distributed over chromosomal segments that include hypomethylated euchromatic regions $[11,12]$. Little is known about the detailed methylation states of the regions around assorted repetitive sequences in plant genomes. We are interested in the methylation state surrounding the various types of plant repetitive sequences.

According to the decoded sequences [13], the rice genome comprises 389 mega base pairs $(\mathrm{Mb})$ in total nucleotide length, and is one of the most compact genomes in monocotyledonous species. Despite its small genome size, TEs account for at least 35\% of the total genome in rice. Representatives from all known TEs exist in the rice genome. The TEs in the rice genome consist mainly of two types of elements categorized into class I and class II, whose contents account for $20 \%$ and $13 \%$, respectively, of the total length of the rice genome. Class I elements, including long terminal-repeat retrotransposons (LTR elements) and non-LTR retrotransposons (longand short-interspersed nucleotide elements: LINEs and SINEs), which transpose in the copy-and-paste manner. In particular, LTR elements are largely localized in gene-poor, heterochromatic regions such as the centromeric and pericentromeric regions [13]. Class II elements, which transpose in the cut-and-paste manner, outnumber class I elements by more than two fold, and often appear in euchromatic regions. The average size of class II elements is less than $300 \mathrm{bp}$, while that of class I elements is greater than 1000 bp, indicating that a large number of class II elements are scattered in the rice genome [13]. These differentially distributed TEs should reside in various differen genomic environments, particularly in terms of DNA methylation.

Previously, Takata et al. [14] detected DNA methylation in the flanking regions of two miniature inverted repeat transposable elements (MITEs) in AA-genome Oryza species using a transposon-display (TD) method. The proportion of methylated fragments in the total number of fragments was clearly different in the two MITEs. The methylation frequency estimated for one of the two MITEs was much lower than the average methylation frequency of the entire genomes of Oryza species. The results implied that MITEs show different preferences of the methylation state in their flanking sequences. This led us to pose the question of whether the sequences around TEs are hypermethylated or in specific methylation states.

In order to address the question, here we performed TD analyses targeting 12 rice TEs, which include class I, II, and other-class elements. The degrees of methylation of the TE flanking sequences were investigated by comparing the TD profiles between MspI and HpaII-digested samples. The 12 TEs tested here were found to show different features with regard to chromosomal distribution, copy number, and degree of conservation within a cognate family. We attempted to find relationships between the methylation state in the sequences adjacent to the TE and copy number or degree of conservation within the cognate family. The individual TE families reside in different genomic segments with varying degrees of methylation, but each family is present in an environment with a particular degree of methylation.

\section{Results and Discussion Selection of rice TEs}

To examine the methylation states of the sequences flanking various repetitive elements, we selected 12 rice TEs that were classified into three groups: class I, class II, and a third group containing unknown type elements (Table $1)$. The representative sequences for the individual TEs examined here were those published in the papers listed in Table 1. Of the five elements in class I, noaCRR1 [15], RIRE5 (Taguchi and Otsubo unpublished result), and RIRE7 [16] belong to the LTR elements, and p-SINE1 [17] is a representative of rice SINEs. Two Ty3/gypsy type elements, noaCRR1 and RIRE7, were distributed around the centromeres. RIRE5, which is a member of the Ty1/copia type elements, was broadly distributed over the rice chromosomes, but not in the centromere. p-SINE1 is small sized (less than $500 \mathrm{bp}$ ) and was distributed randomly in euchromatic regions. 
Table I: Copy numbers and conservation degrees of 12 transposable elements based on the BLAST analyses

\begin{tabular}{|c|c|c|c|c|c|c|c|}
\hline TE & Accession or reference $(\mathrm{I})$ & Size $(\mathrm{Pb})$ & $\begin{array}{l}\text { Total count of } \\
\text { Blast (A) (2) }\end{array}$ & $\begin{array}{l}\text { Highest score } \\
\text { (bit) (3) }\end{array}$ & $\begin{array}{l}\text { Count over } \\
80 \% \text { of the } \\
\text { highest score } \\
\text { (B) (4) }\end{array}$ & $\begin{array}{l}80 \% \text { of highest } \\
\text { score }\end{array}$ & $\begin{array}{c}\text { Conservation } \\
\text { degree } \\
(B / A \%)(5)\end{array}$ \\
\hline noaCRRI & AY827986 & 794 & 479 & 1467 & 29 & 1173 & 6.05 \\
\hline RIRE5 & ABI05803LTR & 995 & 270 & 1674 & 14 & 1339 & 5.19 \\
\hline RIRE7 & AB033234LTR & 858 & 119 & $|54|$ & 30 & 1232 & 25.21 \\
\hline P-SINEI & $\begin{array}{l}\text { Mochizuki et al. (1992) } \\
\text { p-SINEI consensus }\end{array}$ & 123 & 207 & 209 & 66 & 167 & 31.88 \\
\hline Akan & $\mathrm{AB} 077838$ & 486 & 110 & 784 & 47 & 627 & 42.73 \\
\hline Kiddo & AF484680 & 269 & 42 & 459 & 37 & 367 & 88.1 \\
\hline Kiserul & $\mathrm{AB} 077834$ & 259 & 835 & 453 & 390 & 362 & 46.71 \\
\hline mPing & BK000588 & 430 & 50 & 795 & 48 & 636 & 96 \\
\hline Mashu & AB077839 & 263 & 563 & 457 & 441 & 360 & 78.33 \\
\hline Tabitoll & AB07783I & 146 & 563 & 226 & 339 & 180 & 60.21 \\
\hline Toya & $\mathrm{AB} 077842$ & 176 & 11 & 287 & 8 & 229 & 72.73 \\
\hline Basho & Inukai and Sano (2002) MS399 & 201 & 396 & 359 & 29 & 287 & 7.32 \\
\hline
\end{tabular}

I: Representatives of the 12 TEs were selected from these accessions or references, which were used as the queries for the BLAST analyses.

2: These correspond to numbers of the sequences hit by the rice BLAST analyses at NCBI as described in Materials and Methods.

3: The scores are those of the most homologous sequences hit by the BLAST analyses.

4: The numbers of the sequences with more than $80 \%$ of the highest scores.

5: Conservation degrees of the TEs are estimated by percentages of the counts with more than $80 \%$ of the highest scores $(B)$ in the total counts $(A)$.

The Class II elements tested here consisted of Akan [18], Kiddo [19], Kiseru [18], mPing [20-22], Mashu [18], TabitoII [18], and Toya [18], all of which are miniature inverted repeat transposable elements (MITEs). Although mPing and TabitoII are affiliated with the Tourist family, the two structures are markedly different. Toya may constitute a large group of plant TEs, because elements that resemble Toya have been discovered in the tobacco [23] and wheat genomes [24]. For Akan, Kiddo, and Mashu, no counterparts have been identified so far. The class II elements in rice are dominated by the remarkable range of MITEs. The sequences for Basho, belonging to the unknown group [25], are present in certain portions of the rice genome, for instance Turcotte et al. [26] estimated the proportion of the unknown TE-like sequences in $910 \mathrm{~kb}$ of rice genomic DNA sequences to be $1.3 \%$. BLAST searches did not find any specific features of the distribution patterns of the six MITEs and Basho in rice chromosomes, and they appeared to be dispersed over all euchromatic regions (data not shown).

\section{Copy number and conservation of TE sequences}

Copy number is an important factor when one considers the cause of homology-dependent DNA methylation. However, it is difficult to estimate the precise numbers of repetitions even if the complete database is available. This is because most of the repetitive sequences have diversified during evolution. The older elements are more differentiated within the cognate sequences than the new elements. In order to survey the copy number and the degree of sequence conservation for the 12 TEs, we performed BLAST searches specifying the rice genome ([27] as of April, 2007), which takes account of gapped alignments between the query and subject sequences (Table 1 ). The outcomes of the BLAST searches using the representative sequences published for the 12 TEs as the queries revealed the total hit numbers, alignments of the hit sequences, and the bit scores. The total hit numbers of the 12 TEs were widely distributed from 11 to 835 (Table 1 ). We estimated the degrees of conservation in the individual TE families indicating the proportion of the conserved copy that was designated over $80 \%$ of the maximum bit score in the BLAST search using the most representative sequence, i.e., the $10 \%$ conservation degree does not mean $10 \%$ identity, but means that $10 \%$ of the total copy number in the TE family possesses more than $80 \%$ of the maximum bit score. The degree of conservation of the TEs analyzed here varied widely, ranging from $5 \%$ to $96 \%$. Low conservation was seen in noaCRR1, RIRE5, and Basho, while the conserved copies of mPing, which is an active element in rice, accounted for $96 \%$ of the total copy number (Table 1). The information about the copy number and conservation degree was used for consideration of the relationships between DNA methylation and TEs.

Transposon-displays for the cultivated and wild rice strains The detection of methylation in TE flanking sequences was performed by the TD method based on amplified fragment length polymorphisms (AFLP), which is basically similar to that described by Takata et al. [14]. We designed the primers taking account of the conserved region within the 60-bp consensus sequences for the 12 TEs (see Additional file 1). All the 60-bp consensus 
sequences are located in the $5^{\prime}$ end of the TEs. The detection of methylated fragments by TD requires a comparison between DNAs digested with methylation-sensitive and insensitive-restriction enzyme isoschizomers, e.g., HpaII and MspI, which recognize the same tetranucleotide sequence (5'-CCGG-3'). Hence, we surveyed the presence of cytosine methylation at 5'-CCGG-3' sites, which were located upstream of the TE copies. If a fragment from an MspI-digested sample was located at the same position as that from the HpaII-digested sample, the genomic segment was considered to be unmethylated. A fragment was considered to be methylated when the fragment derived from the MspI-digested sample did not correspond to that from the HpaII-digested sample. Nine rice genomes from Japonica, Indica, and O. rufipogon were tested in this study, all of which are closely related to each other, and whose hybrids produce fertile seeds. Evaluation of the nine strains was of benefit to verify the efficacy of the TD method for the detection of genome wide methylation. In the analyses, the same gel profile was reproduced in three independent trials of each primer pair.

Because of the ambiguity of fragments larger than 500 bp detected by the TD method, only fragments below 500 bp were considered valid for evaluation in this study. The TD analyses showed different numbers of bands among the 12 TEs (Figure 1), reflecting the copy number of the TE family and its degree of conservation. In all the TEs examined here except mPing, the numbers of fragments below 500 bp detected in the MspI-digested samples were not significantly different among the nine strains examined (Figure 1). Although the TD for mPing produced 27 - 38 bands below 500 bp in Japonica strains, as expected from

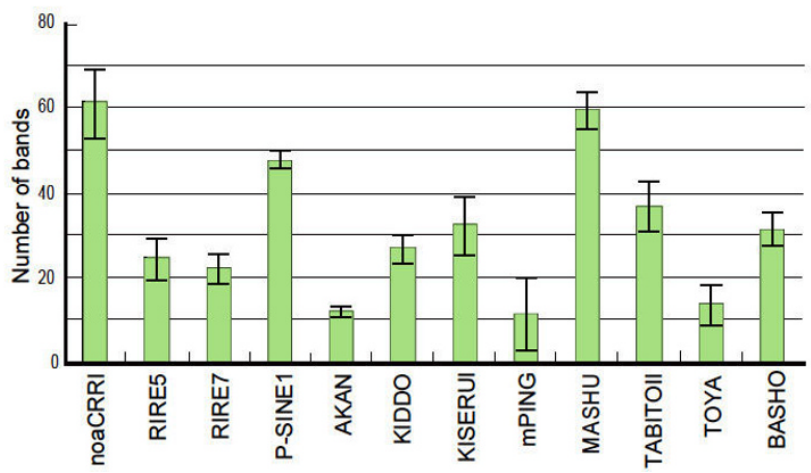

Figure I

Average numbers of bands obtained by TD analyses for the 12 rice TEs. Each column shows the average number of bands that were estimated from the TD analyses of the nine cultivated and wild rice strains. The TD bands smaller than 500 bp in the Mspl-digested samples were counted in this study. Standard deviations were calculated from the data from the nine rice strains. the rice genome database, limited numbers (11-4) of bands for mPing were detectable in Indica and O. rufipogon strains. Indeed, it has been reported that Indica strains possess lower copy numbers of mPing than Japonica strains $[20,21]$.

\section{Detection of DNA methylation}

In order to define the average degrees of cytosine methylation in the whole genomes, the AFLP method was employed using two sets of the digested samples (HpaII/ EcoRI and MspI/EcoRI) for the nine genomes from the cultivated and wild rice strains, which were the same materials as used here. The proportion of methylated fragments in the total number of fragments detected by AFLP ranged between 23\% and 28\% among the nine genomes (Figure 2 ). The results implied that an average of $24 \%$ of the CCGG motifs in the examined genomes were methylated, as the distribution of EcoRI sites was not strongly influenced by methylation.

Comparison of the TD patterns for the HpaII and MspIdigested samples allowed us to estimate the proportions of the methylated fragments flanked by the 5' end of the TEs (see Figure 3 ). Figure 2 shows the average proportions of methylated fragments in the total MspI fragments of the nine strains. The small standard deviations found in noaCRR1, p-SINE1, and Mashu validate the efficacy of the TD method as capable of detecting methylated fragments equivalently in the different strains. The highest proportions of methylated sequences (over 50\%) were observed in the flanking regions of noaCRR1, RIRE5, RIRE7, and Akan. These results were appropriate because the noaCRR1 family and RIRE7 elements are concentrated in the centro-

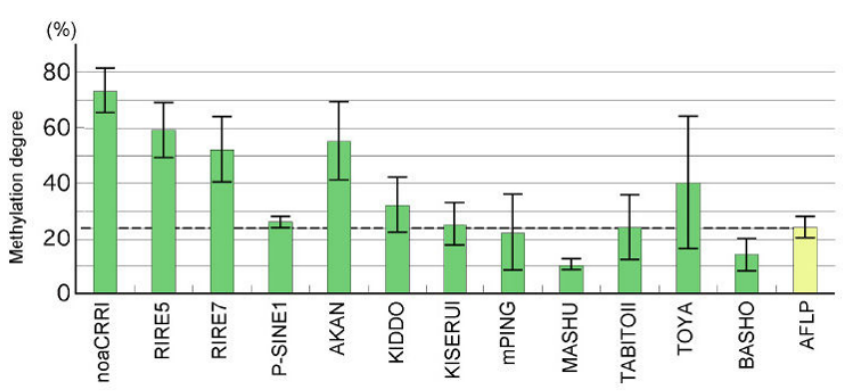

Figure 2

Methylation degrees in the 5 ' flanking sequences of the 12 rice TEs. The TD bands smaller than 500 bp in the Mspl-digested samples were compared with those in the corresponding $\mathrm{H}$ pall-digested samples. Methylation degrees are expressed as the proportion of the methylated bands in the total bands of the Mspl-digested samples. The average methylation degree $(24.3 \%)$ for the whole genomes from the nine strains was estimated by AFLP analyses as described in the Methods, and is indicated by a dotted line. Standard deviations were calculated with the data from the nine rice strains. 

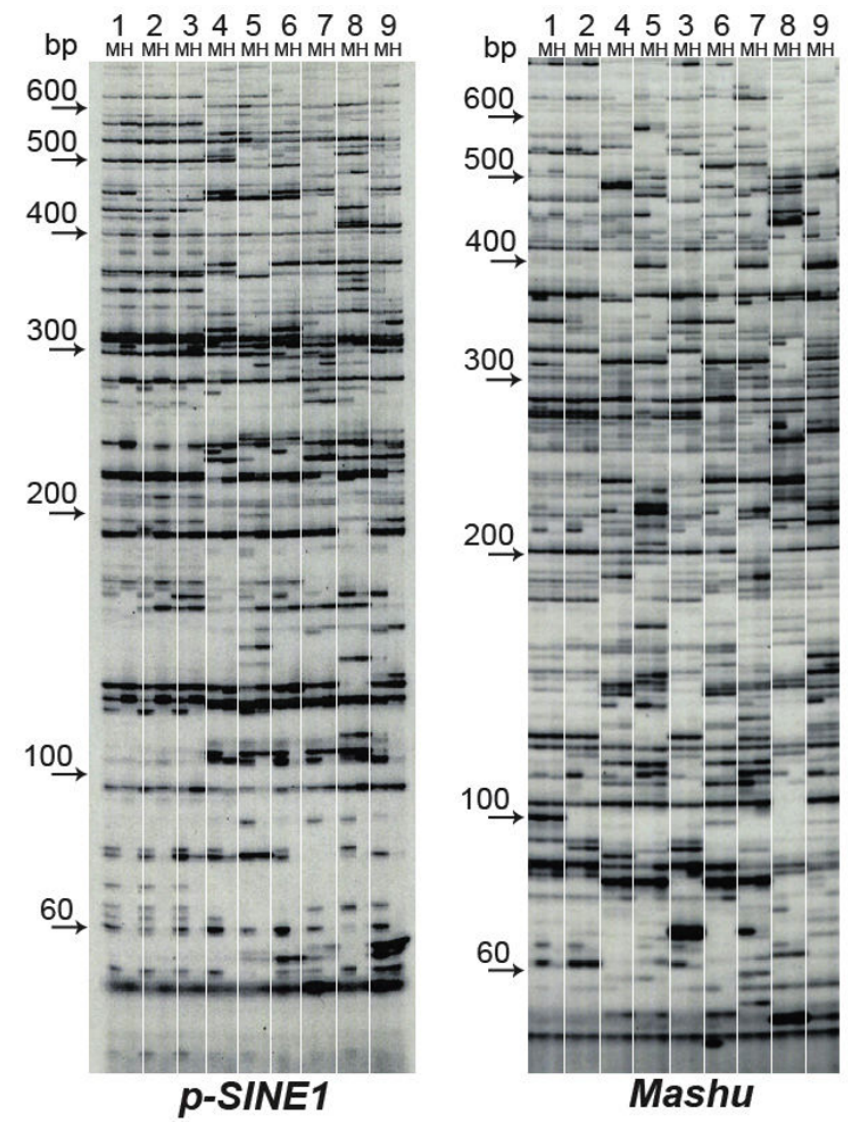

Figure 3

Transposon displays for $p$-SINEI and Mashu in the nine cultivated and wild rice strains. The rice strains consist of A58 (no. I), T65 (no. 2), Nipponbare (no. 3), IR36 (no. 4), Kasalath (no. 5), \#I08 (no. 6), WI07 (no. 7), W593 (no. 8), and W630 (no. 9). In each strain, the two TD samples digested with $\mathrm{Mspl}(\mathrm{M})$ and $\mathrm{Hpall}(\mathrm{H})$ were loaded and electrophoresed in a $50 \%$ polyacrylamide gel. Methylated fragments are denoted by the fact that a fragment of the Mspl-digested sample has no corresponding fragment in the Hpall-digested sample. The sizes from $60 \mathrm{bp}$ to $600 \mathrm{bp}$ are indicated by the arrows.

meric or pericentromeric regions in rice, where the genomic DNA is heavily methylated $[15,16,28]$. The other LTR element, RIRE5, which is not specifically localized in centromeric or pericentromeric regions, showed a similar tendency to the above LTR elements. These results for the LTR elements agreed with those obtained by highthroughput methods that revealed TEs account for a high proportion of the heavily methylated genomic fraction $[5,6,9]$. Akan is the only class II element that resides in heavily methylated fractions that might be related to heterochromatic regions, but it is localized neither in centromeric nor pericentromeric regions according to BLAST searches (data not shown).
In contrast, the flanking sequences of Mashu (Figure 3) and Basho were hypomethylated (under 20\%) in all nine genomes and showed very small standard deviations. Moderately methylated sequences (around 30\%) were found in the flanking sequences of Kiddo and Toya. Those of the copies of p-SINE1, KiseruI, mPing, and TabitoII had similar methylation levels to the average methylation state of the whole genome. Thus, individual TEs reside in chromosomal regions with varying levels of methylation, although stable methylation states of the TE flanking sequences were observed within the same TE family. In the class II elements, the proportions of the methylated fragments differed widely, ranging from that of Akan to that of Mashu. Like noaCRR1 and RIRE7, the other individual TE families were present in particular preferential methylation environments of the Oryza genomes.

\section{Relationships with copy number}

Mashu has more than 500 copies in the rice genome (Table 1 and Figure 3). Despite the large copy number, Mashu is not an old element, because the copy organization is relatively conserved and the insertion sites are highly polymorphic between Japonica and Indica strains [29]. The hypomethylation environment around the copies of Mashu suggests that the copy number of this TE does not influence the methylation state of the adjacent sequences. The sequence of Mashu itself might be associated with the low methylation state, as are the surrounding sequences. It is not necessarily the case that a TE with an abundant copy number is correlated with a high methylation state. TE activities might not always be controlled by DNA methylation, and instead may be controlled in alternative manner(s) such as by gap repair [30,31] or unknown host function(s) to prevent harmful behaviors by TEs. Akan, mPing, and Toya have different methylation states in the surrounding sequences among the nine strains, as shown by the large standard deviations (Figure 2). These are low copy number elements (Figure 1), for which it might be difficult to establish stable methylation states around the elements. Besides the low copy number, the instability of the methylation degree around mPing might be due to some activity of the element, which enables it to be inserted into various methylation environments in the genome. Unfortunately, the other active elements in the rice genome were unsuitable for TD analysis due to their very low copy numbers (Tos17 [32] and Karma [33]) and the presence of a MspI/HpaII site at the terminal of the element ( $n$ Dart $[34,35])$. The other class II elements, which should be immobilized or very low active elements, were embedded in relatively stable methylation states of the genomic sequences.

\section{Relationships with conservation degree}

The degree of conservation is a rough indication of copy divergence in a TE family (Table 1). Old TE family mem- 
bers tend to have a low degree of conservation and divergent copy organization, while new family members might have better-conserved copy constituents. The conserved elements may be active, like mPing, which was the only active class II element in this study [20-22]. Hence, the degree of conservation is certainly related to the activity of the TE element. Here, we tested whether there is a correlation between TE conservation and methylation around the TE. As shown in Table 1 and Figure 2, we did not find a particular correlation between them. This implies an independence of the TE copy organization from the methylation environment in the genome. However, we do not know whether the methylation environment in the genome affect the activity of the TE elements.

\section{Spread of methylation to the adjacent sequences}

When the TD fragments of the MspI-digested samples were sorted into those with distances from the element below $200 \mathrm{bp}$ and above $200 \mathrm{bp}$, the degrees of methylation were found to be different in the proximal and distal regions of p-SINE1 and TabitoII (Figure 3 and 4). These proximal regions showed an even heavier methylation state than the distal regions. It seems likely that the methylation state of the TE has spread to the neighboring sequences after insertion. If so, the methylation state of the TE itself should be linked with that of the adjacent sequences. The higher degrees of methylation of sequences within 200-bp of p-SINE1 and TabitoII might reflect the hypermethylation state of the elements (Figure 4). In fact, $p$-SINE1 copies in the rice genome were strongly suppressed by methylation, whereas p-SINE1 could be transcribed when the methylated state was cancelled by anti-sense genes homologous to Arabidopsis DDM1 or by 5-azacytidine (Ohtsubo et al. unpublished data). In a transgenic rice line retaining a $\beta$-glucuronidase reporter gene, the methylation initially occurred in its promoter region derived from the rice pararetrovirus (rice tungro bacilliform virus: RTBV) and gradually expanded to the $\beta$-glucuronidase reporter coding sequence in subsequent generations of homozygous offspring [36]. In fact, the rice genome contains about 30 methylated sequences homologous to RTBV [37], which might induce methylation from the promoter to the reporter coding sequence. In fission yeast, the spread of heterochromatin requires read-through transcription to generate RNA interference (RNAi) via small interfering RNAs (siRNAs) [38,39]. Similarly, the spread of DNA methylation might require readthrough transcription for the primary substrates. As in the case of the LTR elements where the flanking regions are methylated, the methylated sequence could force methylation of the nearby low-methylated sequences. On the other hand, most of the class II elements examined here were present in low-methylated regions. These elements produce few transcripts corresponding to their genomic sequences, because no well-matched transcripts or ESTs
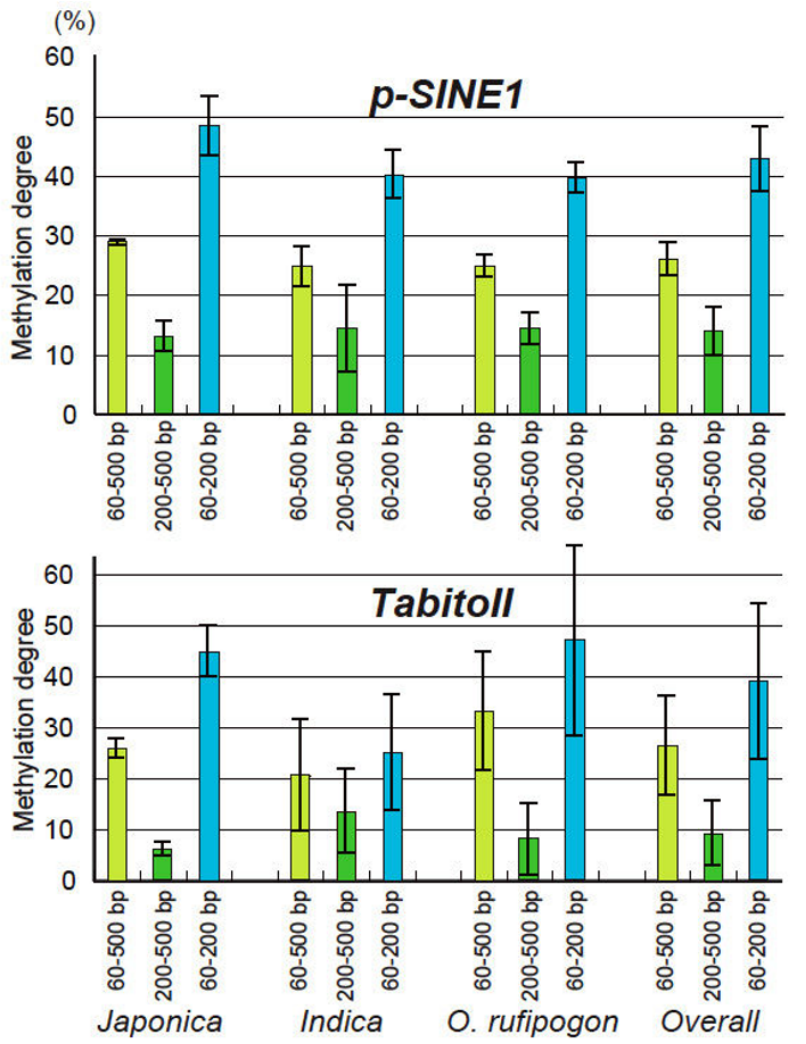

Figure 4

Differential methylation degrees dependent on the distance from the copies of p-SINEI and Tabitoll. Based on the band sizes in the TD profiles of the Mspldigested samples, the fragments were divided into two groups: one ranging from 60 to $200 \mathrm{bp}$ and the other ranging from 200 to $500 \mathrm{bp}$. Methylation degrees were estimated for the fragments in these two ranges and the fragments in the full range from 60 to $500 \mathrm{bp}$. Within a 500-bp range from the elements, the proximal regions of both p-SINEI and Tabitoll are markedly methylated compared with the distal regions. This tendency was also found in the individual rice strains, as illustrated for Japonica (including A58, T65, and Nipponbare), Indica (IR36, Kasalath, and \#I08), and O. rufipogon (WI07, W593, and W630) along with the overall degrees.

were deposited in the databases (data not shown). Therefore, the methylation states of these elements should be as low as their surroundings if methylation is dependent on siRNAs. Alternatively, it is also possible that the methylation states of the surroundings have influenced those of the elements.

\section{TEs characterized by degree of methylation of the surroundings}

Target site preferences consisting of only a few base pairs have been identified in certain types of MITEs [40-42]. However, it is still difficult to predict the insertion sequences of TEs in the genome even if the known flank- 
ing sequences are available, since these sequences show no consensus. However, as shown by Figure 2, TEs are nicely characterized by the degree of methylation of the TE flanking sequences. Although we do not know yet whether a TE can select an insertion site based on the methylation state, our results clearly show that each TE family is characterized by the methylation state of its surroundings. This is the first report demonstrating that TEs in the genome are associated with a particular methylation environment, which is a feature of a given TE.

\section{Conclusion}

TD analysis facilitated a comparison of the methylation degrees among different TEs. In the rice genome database, the 12 TEs analyzed here showed different features of chromosomal distribution, copy number, and conservation degree within a cognate family. The TD analyses revealed that the individual TE families reside in different genomic segments with varying degrees of methylation, but the methylation environments are mostly similar within a given family. The sequences in the vicinity of retrotransposons tend to have a heavier methylation state than those of transposons. A markedly lower methylation state was seen for the flanking sequences of Mashu and Basho. We failed to find a relationship between the copy number of a TE and the methylation state of the adjacent sequences. Our results also detected no correlation between TE conservation and the methylation state around the TE. In the cases of p-SINE1 and TabitoII, the degree of methylation decreased with increasing distance from the elements. If most TEs are methylated, as demonstrated by high-throughput analyses $[5-7,10]$, the methylation state of a TE itself might not be simply associated with that of its surroundings. Our results demonstrate that rice TEs are localized in genomic environments with different degrees of methylation characteristic of the particular TE.

\section{Methods}

\section{BLAST and BLAT analyses}

In order to investigate the copy numbers and degrees of conservation of the 12 TEs, we used the BLAST website [27] specified for the rice genome at NCBI. This BLAST analysis considered gaps between the query and subject. All the queries used were representative sequences that were given accession numbers or were selected from reference papers. The other online genome analysis called the Rice Annotation Project DataBase (RAP-DB) developed by the National Institute of Genetics, Japan, can find perfect sequence matches of 33 bases, and sometimes down to 20 bases. The chromosomal locations of the elements can also be searched for by using RAP-BLAT [43]. This system does not consider gaps between the query and subject.

\section{Plant materials and genomic DNA extraction}

The nine rice strains used in these experiments were as follows: A58, T65, and Nipponbare (Oryza sativa, Japonica), IR36, Kasalath, and 108 (O. sativa, Indica), W107 and W630 (O. rufipogon, annual), and W593 (O. rufipogon, perennial). These strains are closely related species that can be cross-pollinated with each other. They were maintained as inbred strains by several selfings. In order to obtain genomic DNA with uniform genomic environmental conditions for methylation, all the seeds were sown at the same time and the plants were grown for 3 weeks at about $25^{\circ} \mathrm{C}$. Total genomic DNA was isolated from the above-ground parts of the plants (excluding roots), as described by Nagano et al. [18].

\section{Amplified fragment polymorphism (AFLP) and transposon display (TD) to analyze methylation sites}

In order to investigate the average frequencies of the methylation-sensitive sites in the rice and related genomes, we used an AFLP method that was basically similar to that reported by Takata et al. [14]. The isoschizomers HpaII and MspI were used instead of MseI and TaqI as 'frequent cutter' enzymes. Both HpaII and MspI recognize the same tetranucleotide sequence (5'-CCGG-3'), but exhibit differential sensitivity to DNA methylation. HpaII is sensitive to the methylation of any cytosine of 5'CCGG-3', whereas MspI cuts 5'-C5mCGG-3', but not 5'${ }^{5 m}$ CCGG-3'. Thus, 5'-5m CCGG-3' or 5'-5m C ${ }^{5 m}$ CGG-3' at the proximal site of TEs may not give band in this TD method. EcoRI (5'-GAATTC-3') was employed as the 'lessfrequent cutter' enzyme. The oligonucleotides used for adaptors and primers in this study are listed in Additional file 2 . All the adaptors were prepared by annealing the two adaptor primers at room temperature after boiling. DNA digestion, adaptor ligation, and PCR reactions were performed according to the method of Takagi et al. [29]. Two sets of digestion reactions (HpaII/EcoRI and MspI/EcoRI) were carried out simultaneously. Approximately $0.25 \mu \mathrm{g}$ of rice genomic DNA was digested with 5 units of each enzyme in $25 \mu \mathrm{l}$ of a suitable digestion buffer. Each of the HpaII/MspI and EcoRI adaptors $(50 \mu \mathrm{M})$ was ligated to the digested genomic DNA (200 ng) by the addition of $10 \mu \mathrm{l}$ of a mix containing $1 \times$ ligation buffer, $0.5 \mu \mathrm{M}$ ATP, and 1 Weiss unit of T4 DNA ligase and incubation at $16^{\circ} \mathrm{C}$ overnight. The first PCR reaction was performed in $20 \mu \mathrm{l}$ of a reaction mixture containing $1 \times$ PCR buffer, $0.2 \mathrm{mM}$ dNTPs (Takara Shuzo Co Ltd., Osaka, Japan), 15 ng of the adaptor-ligated DNA, 10 pmol of each first primer, and 1 unit of EX Taq polymerase (Takara Shuzo Co Ltd.). The first PCR reaction was carried out as follows: $72^{\circ} \mathrm{C} / 2 \mathrm{~min}$; $94^{\circ} \mathrm{C} / 3 \mathrm{~min} ; 24$ cycles of $94^{\circ} \mathrm{C} / 30 \mathrm{sec}, 54^{\circ} \mathrm{C} / 30 \mathrm{sec}$ and $72^{\circ} \mathrm{C} / 1 \mathrm{~min}$, and a final cycle of $72^{\circ} \mathrm{C} / 5 \mathrm{~min}$. The PCR product was then diluted 20 -fold with sterilized water. Nested primers were used for the second PCR: the 5' end of the second primer was labeled with FITC (lambda 
$\mathrm{em}=495 \mathrm{~nm}$ ), and the $3^{\prime}$ end of the second adaptor primers was overhung by three selected nucleotides (ATC, CTC, GAT, GTT or TTA). These five different types of 3' ends of the second adaptor primers were used for each trial (see Additional file 2). The second PCR reaction was performed with the same reaction mixture as the first PCR plus $2 \mu \mathrm{l}$ of $50 \%$ DMSO. For temperature cycling, we modified the conditions used in the "touchdown method": $94^{\circ} \mathrm{C} / 5 \mathrm{~min}$, then 10 cycles beginning with $94^{\circ} \mathrm{C} / 30 \mathrm{sec}, 68^{\circ} \mathrm{C} / 30 \mathrm{sec}, 72^{\circ} \mathrm{C} / 1 \mathrm{~min}$, followed by a reduction of the annealing temperature of the first cycle $\left(68^{\circ} \mathrm{C}\right)$ with a $1^{\circ} \mathrm{C}$ increment per cycle, and then 27 cycles at an annealing temperature of $58^{\circ} \mathrm{C}$, and a final incubation at $72^{\circ} \mathrm{C} / 5 \mathrm{~min}$. The reaction products were electrophoresed on a $3.5 \%$ denaturing polyacrylamide gel containing $50 \mathrm{ml}$ of a mixture of $3.5 \mathrm{ml}$ of Long Ranger (Takara Shuzo Co Ltd.) (equivalent to $50 \%$ polyacrylamide:bisacrylamide solution $=19: 1)$ and $7.0 \mathrm{M}$ urea. Internal Lane Standard 600 (Promega, Madison, Wisconsin, USA) was used as the size marker from $60 \mathrm{bp}$ to 600 bp, which was labeled with carboxy-X-rhodamine (lambda em $=576 \mathrm{~nm}$ ). After the gel was pre-run using $1 \times$ TBE as running buffer, the samples were electrophoresed at a constant $1700 \mathrm{~V}$ for $80 \mathrm{~min}$. The fragments and size markers were visualized by scanning the fluorescent signals in the gel plate using a Typhoon 8600 scanner (Molecular Dynamics Inc. Sunnyvale, California).

TD analyses were applied for detection of methylation in the flanking sequences of the 12 TEs. The TD basically followed the AFLP method described above with the following modifications. In order to design the primers in the 5' side of the individual TE sequences, we made consensuses for $60 \mathrm{bp}$ of the $5^{\prime}$ end sequences of the individual TEs after surveying sequences homologous to the representative TEs using RAP-BLAT [43] (see Additional file 1). Based on the consensus for each element, nested primers for the TEs were designed, and the 5 ' end of the second TE primer was labeled with FITC (see Additional file 2). No overhung nucleotide was added at the 3 ' end of the second adaptor primers in the TD method. In the cases of LTR elements, the PCR reactions elongate to both the outside and inside of the elements, because the primer sequences reside in the tandem repeats of the LTR elements. A very limited number of the TD bands were directed to the internal sequence of LTR elements due to its higher conservation among the copies compared with the flanking sequences. Thus, we did not take these bands away from the total band numbers when the proportions of methylated bands were calculated.

\section{List of abbreviations \\ AFLP - amplified fragment length polymorphism \\ MITE - miniature inverted repeat transposable element}

LINE - long interspersed nucleotide element

LTR element - long terminal-repeat retrotransposon

SINE - short interspersed nucleotide element

TD - transposon display

TE - transposable element

\section{Authors' contributions}

$\mathrm{MK}, \mathrm{AK}$, and AT conceived the experiments and carried out the molecular analyses and databases searches. YK conceived of the study and drafted the manuscript. HO participated in the sequence alignment, database analysis, and the design of the study. YK and YS participated in the basic design of this study and coordination. All authors read and approved the final manuscript.

\section{Additional material}

\section{Additional file 1}

The 60-bp consensus sequences of the 5' end of 12 TEs. The data provided represent the 60-bp consensus sequences determined from the rice database for the $5^{\prime}$ end of 12 TEs.

Click here for file

[http://www.biomedcentral.com/content/supplementary/1471-

2164-8-469-S1.pdf]

\section{Additional file 2}

Primers used in this study. All the primer sequences used in this study are provided.

Click here for file

[http://www.biomedcentral.com/content/supplementary/1471-

2164-8-469-S2.pdf]

\section{Acknowledgements}

We thank Hironori Nagano, Hokkaido University, for technical suggestions and comments on the manuscript and Yu Taguchi, University of Tokyo, for providing unpublished data. This work was supported by grants from the Ministry of Education, Science, and Culture, Japan.

\section{References}

I. Yoder JA, Walsh CP, Bestor TH: Cytosine methylation and the ecology of intragenomic parasites. Trends Genet 1997, 13(8):335-340.

2. Bender J: DNA methylation and epigenetics. Annu Rev Plant Biol 2004, 55:4I-68.

3. Rangwala SH, Richards EJ: The value-added genome: building and maintaining genomic cytosine methylation landscapes. Curr Opin Genet Dev 2004, I4(6):686-69I.

4. Wong $\mathrm{LH}$, Choo $\mathrm{KH}$ : Evolutionary dynamics of transposable elements at the centromere. Trends Genet 2004, 20(12):6II-616.

5. Zhang X, Yazaki J, Sundaresan A, Cokus S, Chan SW, Chen H, Henderson IR, Shinn P, Pellegrini M, Jacobsen SE, Ecker JR: Genome-wide high-resolution mapping and functional analysis of DNA methylation in arabidopsis. Cell 2006, $126(6): 1189-1201$.

6. Zilberman D, Gehring M, Tran RK, Ballinger T, Henikoff S: Genomewide analysis of Arabidopsis thaliana DNA methylation 
uncovers an interdependence between methylation and transcription. Nat Genet 2007, 39(I):6I-69.

7. Rabinowicz PD, Citek R, Budiman MA, Nunberg A, Bedell JA, Lakey N, O'Shaughnessy AL, Nascimento LU, McCombie WR, Martienssen RA: Differential methylation of genes and repeats in land plants. Genome Res 2005, I 5(I 0): | 43 |-| 440.

8. Vaughn MW, Tanurd Ic M, Lippman Z, Jiang H, Carrasquillo R, Rabinowicz PD, Dedhia N, McCombie WR, Agier N, Bulski A, Colot V, Doerge RW, Martienssen RA: Epigenetic Natural Variation in Arabidopsis thaliana. PLoS Biol 2007, 5(7):el74.

9. Rabinowicz PD, Palmer LE, May BP, Hemann MT, Lowe SW, McCombie WR, Martienssen RA: Genes and transposons are differentially methylated in plants, but not in mammals. Genome Res 2003, I 3(I 2):2658-2664.

10. Tran RK, Zilberman D, de Bustos C, Ditt RF, Henikoff JG, Lindroth AM, Delrow J, Boyle T, Kwong S, Bryson TD, Jacobsen SE, Henikoff $S$ : Chromatin and siRNA pathways cooperate to maintain DNA methylation of small transposable elements in Arabidopsis. Genome Biol 2005, 6(I I):R90.

11. Mao L, Wood TC, Yu Y, Budiman MA, Tomkins J, Woo S, Sasinowsk M, Presting G, Frisch D, Goff S, Dean RA, Wing RA: Rice transposable elements: a survey of 73,000 sequence-tagged-connectors. Genome Res 2000, 10(7):982-990.

12. Arabidopsis_Genome_Initiative: Analysis of the genome sequence of the flowering plant Arabidopsis thaliana. Nature 2000, 408(68|4):796-8I5.

13. International_Rice_Genome_Sequencing_Project: The map-based sequence of the rice genome. Nature 2005, 436(7052):793-800.

14. Takata M, Kishima Y, Sano Y: DNA methylation polymorphisms in rice and wild rice strains: Detection of epigenetic markers. Breeding Science 2005, 55(I):57-63.

I5. Nagaki K, Neumann P, Zhang D, Ouyang S, Buell CR, Cheng Z, Jiang J: Structure, divergence, and distribution of the CRR centromeric retrotransposon family in rice. Mol Biol Evol 2005, 22(4):845-855.

16. Kumekawa N, Ohmido N, Fukui K, Ohtsubo E, Ohtsubo H: A new gypsy-type retrotransposon, RIRE7: preferential insertion into the tandem repeat sequence Trs $D$ in pericentromeric heterochromatin regions of rice chromosomes. Mol Genet Genomics 200I, 265(3):480-488.

17. Mochizuki K, Umeda M, Ohtsubo H, Ohtsubo E: Characterization of a plant SINE, p-SINEI, in rice genomes. Jpn / Genet 1992, 67(2): $155-166$.

18. Nagano H, Kunii M, Azuma T, Kishima Y, Sano Y: Characterization of the repetitive sequences in a $200-\mathbf{k b}$ region around the rice waxy locus: diversity of transposable elements and presence of veiled repetitive sequences. Genes Genet Syst 2002, 77(2):69-79.

19. Yang G, Dong J, Chandrasekharan MB, Hall TC: Kiddo, a new transposable element family closely associated with rice genes. Mol Genet Genomics 200I, 266(3):4I7-424

20. Jiang N, Bao Z, Zhang $X$, Hirochika H, Eddy SR, McCouch SR, Wessler SR: An active DNA transposon family in rice. Nature 2003, 42 I(6919): 163-167.

21. Kikuchi K, Terauchi K, Wada M, Hirano HY: The plant MITE mPing is mobilized in anther culture. Nature 2003, 42I(6919): $167-170$

22. Nakazaki T, Okumoto Y, Horibata A, Yamahira S, Teraishi M, Nishida $\mathrm{H}$, Inoue $\mathrm{H}$, Tanisaka $\mathrm{T}$ : Mobilization of a transposon in the rice genome. Nature 2003, 42 I (69 I9): I70-172.

23. Schenke D, Sasabe M, Toyoda K, Inagaki YS, Shiraishi T, Ichinose $Y$ : Genomic structure of the NtPDRI gene, harboring the two miniature inverted-repeat transposable elements, NtToya I and NtStowaway 10 I. Genes Genet Syst 2003, 78(6):409-4I8.

24. Saito $M$, Yonemaru J, Ishikawa G, Nakamura T: A candidate autonomous version of the wheat MITE Hikkoshi is present in the rice genome. Mol Genet Genomics 2005, 273(5):404-4I4.

25. Inukai T, Sano Y: Sequence rearrangement in the AT-rich minisatellite of the novel rice transposable element Basho. Genome 2002, 45(3):493-502.

26. Turcotte K, Srinivasan S, Bureau T: Survey of transposable elements from rice genomic sequences. Plant J 2001 25(2):169-179.

27. BLAST Plant genomes [http://www.ncbi.nlm.nih.gov/BLAST/ Genome/PlantBlast.shtml?!0]
28. Mizuno H, Ito K, Wu J, Tanaka T, Kanamori H, Katayose Y, Sasaki T, Matsumoto $\mathrm{T}$ : Identification and mapping of expressed genes, simple sequence repeats and transposable elements in centromeric regions of rice chromosomes. DNA Res 2006, 13(6):267-274

29. Takagi K, Nagano H, Kishima Y, Sano Y: MITE-transposon display efficiently detects polymorphisms among the Oryza AAgenome species. Breeding Science 2003, 53(2): 125-132.

30. Rubin E, Levy AA: Abortive gap repair: underlying mechanism for Ds element formation. Mol Cell Biol 1997, I7( I I):6294-6302.

31. Yamashita S, Takano-Shimizu T, Kitamura K, Mikami T, Kishima Y: Resistance to gap repair of the transposon Tam3 in Antirrhinum majus: a role of the end regions. Genetics 1999, I53(4): $1899-1908$.

32. Hirochika $\mathrm{H}$, Sugimoto $\mathrm{K}$, Otsuki $\mathrm{Y}$, Tsugawa $\mathrm{H}$, Kanda M: Retrotransposons of rice involved in mutations induced by tissue culture. Proc Natl Acad Sci USA 1996, 93( 15):7783-7788.

33. Komatsu M, Shimamoto K, Kyozuka J: Two-step regulation and continuous retrotransposition of the rice LINE-type retrotransposon Karma. Plant Cell 2003, I 5(8): 1934-1944.

34. Tsugane K, Maekawa M, Takagi K, Takahara H, Qian Q, Eun CH, lida $S$ : An active DNA transposon $n$ Dart causing leaf variegation and mutable dwarfism and its related elements in rice. Plant J 2006, 45(I):46-57.

35. Fujino $\mathrm{K}$, Sekiguchi $\mathrm{H}$, Kiguchi $\mathrm{T}$ : Identification of an active transposon in intact rice plants. Mol Genet Genomics 2005, 273(2): $150-157$.

36. Kloti A, He X, Potrykus I, Hohn T, Futterer J: Tissue-specific silencing of a transgene in rice. Proc Natl Acad Sci USA 2002, 99(16): $10881-10886$

37. Kunii M, Kanda M, Nagano H, Uyeda I, Kishima Y, Sano Y: Reconstruction of putative DNA virus from endogenous rice tungro bacilliform virus-like sequences in the rice genome: implications for integration and evolution. BMC Genomics 2004, 5(I):80.

38. Irvine DV, Zaratiegui M, Tolia NH, Goto DB, Chitwood DH, Vaughn MW, Joshua-Tor L, Martienssen RA: Argonaute slicing is required for heterochromatic silencing and spreading. Science 2006, 3 I3(5790): I |34-। I37.

39. Volpe TA, Kidner C, Hall IM, Teng G, Grewal SI, Martienssen RA: Regulation of heterochromatic silencing and histone $\mathrm{H}_{3}$ lysine-9 methylation by RNAi. Science 2002, 297(5588): 1833-1837.

40. Bureau TE, Wessler SR: Stowaway: a new family of inverted repeat elements associated with the genes of both monocotyledonous and dicotyledonous plants. Plant Cell 1994, 6(6):907-916

4I. Bureau TE, Ronald PC, Wessler SR: A computer-based systematic survey reveals the predominance of small invertedrepeat elements in wild-type rice genes. Proc Natl Acad Sci USA 1996, 93( I 6):8524-8529.

42. Naito K, Cho E, Yang G, Campbell MA, Yano K, Okumoto Y, Tanisaka $T$, Wessler SR: Dramatic amplification of a rice transposable element during recent domestication. Proc Natl Acad Sci USA 2006, I03(47): 17620-17625.

43. RAP-DB: rice annotation project database [http:// rapdb.dna.affrc.go.jp/tools/blat]

Publish with Biomed Central and every scientist can read your work free of charge

"BioMed Central will be the most significant development for disseminating the results of biomedical research in our lifetime. "

Sir Paul Nurse, Cancer Research UK

Your research papers will be:

- available free of charge to the entire biomedical community

- peer reviewed and published immediately upon acceptance

- cited in PubMed and archived on PubMed Central

- yours - you keep the copyright 